\title{
A Population Based Hybrid Metaheuristic for the $p$-median Problem
}

\author{
Wayne Pullan
}

\begin{abstract}
The $p$-median problem is one of choosing $p$ facilities from a set of candidates to satisfy the demands of $n$ clients such that the overall cost is minimised. In this paper, PBS, a population based hybrid search algorithm for the $p$ median problem is introduced. The PBS algorithm uses a genetic algorithm based meta-heuristic, primarily based on cut and paste crossover operators, to generate new starting points for a hybrid local search. For larger $p$-median instances, PBS is able to effectively utilise a number of computer processors. It is shown empirically that PBS is able to effectively solve $p$-median problems for a large range of the commonly used $p$-median benchmark instances.
\end{abstract}

\section{INTRODUCTION}

The $p$-median problem (PM) calls for finding the $p$ facilities which minimise the total cost of servicing $n$ clients, where the pair-wise cost of servicing each client from all facilities is given. Each client is only serviced by a single facility and services to clients are not combined. Formally, the $p$-median problem can be stated as [1]:

$$
\begin{aligned}
& \min \sum_{i=1}^{n} \sum_{j=1}^{n} w_{i} d_{i j} x_{i j} \\
& \text { such that: } \sum_{j=1}^{n} x_{i j}=1 \quad \forall i \\
& x_{i j} \quad \leq y_{j} \quad \forall i, j \\
& \sum_{j=1}^{n} y_{j}=p \text {, } \\
& x_{i j} \quad=0 \text { or } 1 \quad \forall i, j \\
& y_{j} \quad=0 \text { or } 1 \quad \forall j
\end{aligned}
$$

where: $n=$ total number of clients, $x_{i j}=1$ if client $i$ assigned to facility $j$ and 0 otherwise, $y_{j}=1$ if facility $j$ exists and 0 otherwise, $w_{i}=$ demand of client $i, d_{i j}=$ distance between client $i$ and facility $j, p=$ number of facilities.

$\mathrm{PM}$ is a prominent combinatorial optimisation problem from location science [2] and cluster analysis [3] and has been shown to be $N P$-hard [4]. Exact methods for solving PM problems include those based on linear programming [5], [6], [7] and dual-based algorithms [6], [8]. However, as with most combinatorial problems, exact methods are limited in the size of the problem that they can deal with so there has also been a focus on heuristic approaches to solving the $p$-median problem. including Variable Neighbour Decomposition Search (VNDS) [9] which was derived from Variable Neighbour Search (VNS) [10], a cooperative parallel variable neighbourhood search (CNVNS) [11] which has been successful on very large problems, Local Optimization (LOPT) [12], Decomposition Procedure (DEC) [12],

Wayne Pullan is with the School of Information and Communication Technology, Griffith University, Gold Coast, QLD, Australia
Lagrangean-Surrogate Heuristic (LSH) [13], Column Generation with Lagrangean-Surrogate Relaxation (CGLS) [14] and a genetic algorithm, ADE [15]. A more recent hybrid heuristic, HYBRID [16], is a multi-start hybrid heuristic that combines elements of several traditional meta-heuristics, and finds near-optimal solutions for a large range of $p$-median instances.

In this work, a population based, hybrid heuristic for the $p$ median problem, dubbed PBS, is introduced. PBS uses a genetic algorithm based meta-heuristic, primarily based on cut and paste crossover operators, to generate new starting points for a hybrid local search. For larger $p$-median instances, PBS is able to effectively utilise a number of computer processors. Based on extensive computational experiments, this study demonstrates that PBS has excellent performance, with regard to robustness and quality of results, on a broad range of widely studied PM benchmark instances, and hence is an effective heuristic for solving PM problems.

The remainder of this article is structured as follows. The PBS algorithm is first described. Next, empirical performance results are presented that establish PBS as an effective metaheuristic for PM solving. This is followed by a more detailed investigation of factors determining PBS performance and, finally, the main contributions of this work are summarised and some directions outlined for future research.

\section{The PBS AlgorithM}

The PBS algorithm is based on the fundamental concept of augmenting a population based meta-heuristic with a Hybrid Local Search (HLS). The role of the meta-heuristic is to provide a wide range of starting points for the HLS while the role of the HLS is to effectively explore regions that are relatively close to these starting points. Key features of the PBS algorithm are now described using the following terminology and syntax: $G=(V, E)$ - a directed or undirected graph, where $V$ is the set of all $n$ vertices and $E$ the set of all possible edges; ' $p$-median solution' - a subset of $V$; 'facility' - a vertex that is in the current $p$-median solution; $p$ - required number of facilities in a $p$-median solution; ' $p$-median cost' - total cost of servicing all $(n-p)$ vertices in $G$ from the $p$ facilities in the $p$-median solution. Each vertex is serviced by a single facility and the cost of servicing all vertices from all facilities is pre-defined for each instance; $P$ - PBS population of $p$-median solutions; $P_{i}-$ a $p$-median solution within the population $P ; C$ - a child $p$-median solution that is currently being created; $|C|-$ the number of facilities currently in $C$; and, $N_{p}$ - the number of computer processors to be used by PBS. 
PBS uses a relatively small population $P$ (of size 8 for a single processor version of PBS and 12 for multiprocessor versions) of $p$-median solutions to provide inputs to genetic crossover and mutation operators. The outputs from these operators subsequently become inputs to the HLS which produces candidate solutions for addition to $P$. At the commencement of PBS, all the initial solutions in $P$ are created by setting each $P_{i}$ to a single vertex chosen uniformly at random from the given graph $G$. Then the HLS is invoked to first increase the number of facilities in $P_{i}$ to $p$ after which an iterative improvement phase is entered and facilityvertex swaps are performed for some number of iterations. Once an initial population has been created, PBS repeatedly performs generations during which all possible mutations and crossovers within $P$ are executed with immediate updating of the population whenever a lower cost $p$-median solution is found. At the completion of each crossover and mutation operation, child solutions $C$ are locally optimised using HLS which, if $|C|=p$ iteratively removes a facility and replaces it with the vertex which results in the largest decrease in the cost of $C$. If $|C|<p$ then the HLS simply adds the vertex which results in the largest decrease in the cost of $C$. To maintain diversity in the population, no new $p$-median solution $C$ is added to $P$ if it is close (in cost) to any $P_{i}$ in $P$. If $N_{p}>1$, PBS operates in a master-slave mode where the master task controls the allocation of pairs of solutions from $P$ to each slave task and the updating of the population $P$. The slave tasks perform the crossover and mutation operators and the subsequent HLS before passing all resulting child solutions back to the master task for possible addition to $P$.

\section{A. Mutation Operator}

The single PBS mutation operator takes a $p$-median solution $P_{i}$ and constructs a child solution $C$ by first randomly selecting some number $q$ in the range $p / 2, \ldots, p$ and then adding $q$ randomly selected facilities from $P_{i}$ and $p-q$ randomly selecting vertices from $G$ to $C$. The HLS is then invoked using $C$ as the starting point and the subsequent, resulting $p$-median solution becomes a candidate to be added to $P$. This operator is very similar to the shaking step of VNS [10].

\section{B. Crossover Operators}

All crossover operators in PBS take two parent $p$-median solutions from $P$ and produce either one or two child solutions. The crossover operators are basically of two types, those that operate in the genotype space and those that function in the phenotype space. The genotype crossover operators $\left(\mathcal{X}_{1}, \mathcal{X}_{2}\right.$ below) and one of the phenotype operators ( $\mathcal{X}_{3}$ below) are the only crossover operators used when $G$ has been specified as a distance matrix while all crossover operators are used when $G$ has been specified using the $(x, y)$ coordinates of the vertices.

The specific crossover operators implemented within PBS are: $\mathcal{X}_{1}$, which constructs a single child solution of $p$ facilities using those facilities in the parent solutions that have the lowest attributable cost to attributable count ratio. The attributable cost of a facility is defined as the component of the total cost of the solution that belongs to the facility and the attributable count is the number of vertices serviced by the facility; $\mathcal{X}_{2}$, which constructs a single child solution by randomly selecting $p$ facilities from the two parents; $\mathcal{X}_{3}$, which first randomly selects two vertices and a random number $q \in[0.1, \ldots, 0.9]$. If $d_{i 1}$ is the distance between facility $i$ and the first selected vertex and $d_{i 2}$ the distance between facility $i$ and the second selected vertex then the first child solution is constructed by combining all facilities $i$ in the first parent where $d_{i 1} / d_{i 2} \leq q$ with all facilities $j$ in the second parent where $d_{j 1} / d_{j 2}>q$. The second child solution is built by combining all facilities $i$ in the first parent where $d_{i 1} / d_{i 2}>q$ with all facilities $j$ in the second parent where $d_{j 1} / d_{j 2} \leq q ; \mathcal{X}_{4}$ uses a randomly generated vertical / horizontal line on the $(x, y)$ plane to divide each parent into two partitions and constructs child solutions by combining opposing partitions from each parent; $\mathcal{X}_{5}$ uses two randomly generated lines on the $(x, y)$ plane to divide each parent into 4 partitions and constructs two child solutions by selecting four distinct partitions, a maximum of three from each parent; $\mathcal{X}_{6}$ uses a randomly generated line on the $(x, y)$ plane to divide each parent into two partitions and the child solutions are constructed as for $\mathcal{X}_{4}$; and $\mathcal{X}_{7}$, which defines a randomly generated rectangle on the $(x, y)$ plane within $G$ and constructs two child solutions by combining partitions from each parent that lie within / outside the rectangle. For all these crossover operators, if more than $p$ facilities are added to a child solution then the required number are randomly removed to reduce the total down to $p$. If less than $p$ facilities are present then the subsequent HLS will add the required number to bring the child solution back to $p$ facilities.

\section{Hybrid Local Search}

The HLS component of PBS takes a $p$-median solution $C$ and, if $|C|<p$, adds facilities to $C$ using a greedy [17] selection (ties broken randomly). Once $|C|=p$, the HLS enters a swap phase where, for each iteration of the HLS, a facility is removed from $C$ and replaced by one that has not previously been a member of $C$. Selection of the facility to be removed from $C$ is, with probability 0.1 , uniformly random (c.f. [18]) and, with probability 0.9 , the facility that has been a member of $C$ the longest (ties broken randomly). Selection of the vertex to be added to $C$ is, with probability 0.1 , uniformly random from the vertices that will cause an decrease in the cost of $C$ and, with probability 0.9 , the vertex that will cause the largest decrease in the cost of $C$ (ties broken randomly). To prevent the HLS cycling around vertices, a tabu [19] mechanism is used which prevents a vertex that has been a member of $C$ from being reselected during the current invocation of the HLS. If, at the completion of the facility-vertex swap there is not an overall improvement in the cost of $C$ then the swap is reversed. The termination criteria for the HLS is either 2,000 iterations or $n / 8$ consecutive swap reversals or the target cost is achieved. 


\section{EMPIRICAl PERFormance Results}

In order to evaluate the performance and behaviour of PBS, extensive computational experiments were performed on a benchmark of commonly used PM instances (in this context, a PM instance is defined as the combination of a graph $G$ and a particular $p$ value). The benchmark consisted of 40 instances from the ORLIB [5] class, three instances from the SL [13] class, 16 instances from the GR [20] class, 28 instances from the RW [16] class and 84 instances from the TSPLIB [21] class giving a total of 171 instances. Instances of this benchmark have between 100 and 11849 vertices and $p$ lies in the range $2, \ldots, 1500$.

All experiments for this study were performed on a Linux cluster computer that, when executing the DIMACS Machine Benchmark (dmclique, ftp://dimacs.rutgers.edu in directory /pub/dsj/clique) on a single computer processor, required $0.31 \mathrm{CPU}$ seconds for $\mathrm{r} 300.5,1.93 \mathrm{CPU}$ seconds for $\mathrm{r} 400.5$ and 7.35 CPU seconds for r500.5. Note that, as PBS is able to execute using a number of computer processors, in the following sections, PBS- $n$ denotes an $n$ computer processor instance of PBS.

In the following section, results from a series of experiments that were aimed at providing a detailed assessment of the performance of PBS are first presented.

\section{A. PBS Performance}

To evaluate the performance of PBS on the instances identified above, extended trials were first performed on the instances where an optimal $p$-median cost was unknown to determine if PBS could improve on the 'best known cost' reported to date in the literature. Then, some number (100 or 9) of independent trials (each of upto 200 generations) of PBS were performed for each instance, using a target $p$-median cost either identical to or close to the respective optimal or best known cost. The results from these experiments are displayed in Table I through Table VIII which utilise the reporting method of [16] (and others) where the optimal or best known cost, the source of the optimal / best known cost, the median cost over the trials for each instance, the percentage error of the average cost over the trials from the optimal or best known cost specified in the table and the average of the PBS CPU / elapsed times until the target $p$ median cost was found (or, if not found, the CPU / elapsed time at which the lowest $p$-median cost solution was located) for each instance are documented. It should be noted that for PBS the times do not include the initial reading of input data files nor the creation of static data structures.

PBS-1 finds the optimal solutions with a success rate of $100 \%$ for all instances of the ORLIB class (Table I), the SL class (Table II) and the GR class (Table III). For the 28 instance RW class (Table IV), PBS-1 found eight improvements on the best known costs in addition to finding all remaining best known costs (all $R W 100$ instances were solved in less than $0.01 \mathrm{CPU}$ seconds). For the $f 1400$ class (Table V), with 18 instances, PBS found eight new best known costs. For the ten remaining instances, the average percentage cost error was 0.002 . For the pcb3038 class (Table VI), with 28 instances, PBS found 22 new best known costs and all six of the remaining currently best known costs. For the rl5934 class (Table VII), with 28 instances, PBS found 24 new best known costs, three of the currently best known costs and had a percentage cost error less than 0.001 on the remaining instance. Finally, for the rl11849 class (Table VIII), with 10 instances, PBS found 10 new best known costs. These results, over a range of different types of instances, support the claim that PBS is robust and also capable of obtaining high quality results.

With regard to the scaling of PBS as the number of processors is increased, using the average elapsed time $T$ for PBS-10 as the base, from Table V through Table VIII the average elapsed time for PBS-20, averaged over all $f 1400$, pcb3038, rl5934 and rl11849 instances, was $0.53 T$ and, for PBS-30, 0.40T.

\begin{tabular}{|c|c|c|c|c|c|c|c|c|c|c|}
\hline \multicolumn{2}{|c|}{ Instance } & \multicolumn{3}{|c|}{ PBS-1 } & \multicolumn{3}{|c|}{ Instance } & \multicolumn{3}{|c|}{ PBS-1 } \\
\hline Name & $\begin{array}{ll}\mathrm{p} & \text { OPT }\end{array}$ & MED & \%ERR & Time & Name & $\mathrm{p}$ & OPT & MED & \%ERR & Time \\
\hline pmed01 & 55819 & 5819 & 0.000 & $\overline{<\epsilon}$ & pmed21 & 5 & 9138 & 9138 & $\overline{0.000}$ & $<\epsilon$ \\
\hline pmed02 & 104093 & 4093 & 0.000 & $<\epsilon$ & pmed22 & 10 & 8579 & 8579 & 0.000 & 0.02 \\
\hline & 104250 & 4250 & 0.000 & $<\epsilon$ & ned 23 & 50 & 4619 & 4619 & 0.000 & 0.01 \\
\hline pmed04 & 203034 & 3034 & 0.000 & $<\epsilon$ & pmed24 & 100 & 2961 & 2961 & 0.000 & 0.06 \\
\hline pmed05 & 331355 & 1355 & 0.000 & $<\epsilon$ & pmed25 & 167 & 1828 & 1828 & 0.000 & 0.39 \\
\hline pmed06 & 57824 & 7824 & 0.000 & $<\epsilon$ & pmed 26 & 5 & 9917 & 9917 & 0.000 & 0.01 \\
\hline pmed07 & 105631 & 5631 & 0.000 & $<\epsilon$ & pmed27 & 10 & 8307 & 8307 & 0.000 & 0.02 \\
\hline pmed08 & 204445 & 4445 & 0.000 & $<\epsilon$ & pmed 28 & 60 & 4498 & 4498 & 0.000 & 0.07 \\
\hline pmed09 & 402734 & 2734 & 0.000 & 0.08 & pmed 29 & 120 & 3033 & 3033 & 0.000 & 0.32 \\
\hline pmed10 & 671255 & 1255 & 0.000 & $<\epsilon$ & pmed 30 & 200 & 1989 & 1989 & 0.000 & 1.89 \\
\hline pmed11 & 57696 & 7696 & 0.000 & $<\epsilon$ & pmed31 & & 10086 & 10086 & 0.000 & 0.01 \\
\hline pmed 12 & 106634 & 6634 & 0.000 & $<\epsilon$ & pmed32 & 10 & 9297 & 9297 & 0.000 & 0.01 \\
\hline & 304374 & 4374 & 0.000 & $<\epsilon$ & pmed33 & 70 & 4700 & 4700 & 0.000 & 0.37 \\
\hline pmed14 & 602968 & 2968 & 0.000 & 0.02 & pmed34 & 140 & 3013 & 3013 & 0.000 & 1.30 \\
\hline pmed 15 & 1001729 & 1729 & 0.000 & 0.56 & pmed35 & & 10400 & 10400 & 0.000 & 0.01 \\
\hline pmed16 & 58162 & 8162 & 0.000 & $<\epsilon$ & pmed36 & 10 & 9934 & 9934 & 0.000 & 0.03 \\
\hline pmed17 & 106999 & 6999 & 0.000 & 0.01 & pmed37 & 80 & 5057 & 5057 & 0.000 & 0.28 \\
\hline & 404809 & 4809 & 0.000 & 0.06 & ed 38 & & 11060 & 11060 & 0.000 & 0.02 \\
\hline pmed19 & 802845 & 2845 & 0.000 & 0.80 & pmed39 & 10 & 9423 & 9423 & 0.000 & 0.01 \\
\hline pmed20 & 1331789 & 1789 & 0.000 & 0.03 & pmed40 & 90 & 5128 & 5128 & 0.000 & 2.87 \\
\hline
\end{tabular}
TABLE I

PBS-1 RESULTS, FROM 100 INDEPENDENT TRIALS, FOR THE CLASS ORLIB [5]: OPTIMAL COST (OPT); MEDIAN COST (MED); AVERAGE PERCENTAGE COST ERROR (\%ERR); AND AVERAGE CPU TIMES IN SECONDS (Time). AVERAGE CPU TIMES $<0.01$ ARE SHOWN AS ' $<\epsilon$ '.

\begin{tabular}{ccccccc}
\hline \multicolumn{3}{c}{ Instance } & & \multicolumn{3}{c}{ PBS-1 } \\
\cline { 1 - 5 } \cline { 5 - 6 } Name & $p$ & OPT & & MED \%ERR Time \\
\hline s1700 233 & 1847 & & 1847 & 0.000 & 0.45 \\
s1800 267 & 2026 & & 2026 & 0.000 & 4.89 \\
s1900 300 & 2106 & 2106 & 0.000 & 3.85 \\
\hline \multicolumn{3}{c}{ TABLE II }
\end{tabular}

PBS-1 RESULTS, FROM 100 INDEPENDENT TRIALS, FOR THE CLASS SL [13]: OPTIMAL COST (OPT); MEDIAN COST (MED); AVERAGE PERCENTAGE COST ERROR (\%ERR); AND AVERAGE CPU TIMES IN SECONDS (TIME).

\section{B. Comparative Results}

The results reported in the previous section clearly demonstrate that PBS achieves excellent performance on standard benchmark instances. However, a comparative analysis of these results, as compared to the results found in the literature for other state-of-the-art PM algorithms, is not a straightforward task as comparisons must take into account both the 


\begin{tabular}{|c|c|c|c|c|c|c|c|c|c|c|}
\hline \multicolumn{2}{|c|}{ Instance } & \multicolumn{3}{|c|}{ PBS-1 } & \multicolumn{3}{|c|}{ Instance } & \multicolumn{3}{|c|}{ PBS-1 } \\
\hline Name & $\begin{array}{ll}\mathrm{p} & \mathrm{OPT}\end{array}$ & $\overline{M E D}$ & \%ERR & Time & Name & 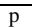 & OPT & MED & \%ERR & Tin \\
\hline gr100 & 55703 & 5703 & 0.000 & $<\epsilon$ & gr150 & 5 & 10839 & 10839 & 0.000 & $\overline{<\epsilon}$ \\
\hline & 104 & 4426 & 0.000 & 0.01 & & 10 & 29 & 729 & 0.000 & 0.01 \\
\hline & 153 & 38 & 0.000 & $<$ & & 15 & 0 & 390 & 0.000 & 0.02 \\
\hline & 203565 & 3565 & 0.000 & 0.02 & & 20 & 6 & 6454 & 0.000 & 0 . \\
\hline & 253 & 3 & 00 & 0.02 & & 25 & 5 & 75 & 00 & 0.09 \\
\hline & 303 & 3032 & 0.000 & 0.01 & & 30 & 5495 & 5495 & 0.000 & 0.08 \\
\hline & 402542 & 2542 & 0.000 & 0.02 & & 40 & 4907 & 4907 & 0.000 & 0.03 \\
\hline & 502083 & 2083 & 0.000 & $<\epsilon$ & & 50 & 4374 & 4374 & 0.000 & 0.50 \\
\hline
\end{tabular}

TABLE III

PBS-1 RESULTS, FROM 100 INDEPENDENT TRIALS, FOR THE CLASS GR [20]: OPTIMAL COST (OPT); MEDIAN COST (MED); AVERAGE PERCENTAGE COST ERROR (\%ERR); AND AVERAGE CPU TIMES IN SeConds (Time). Average CPU times $<0.01$ are Shown As ' $<\epsilon$ '.

\begin{tabular}{|c|c|c|c|c|c|c|}
\hline \multicolumn{4}{|c|}{ Instance } & \multicolumn{3}{|c|}{ PBS-1 } \\
\hline Name & $\mathrm{p}$ & Best & Source & MED & \%ERR & Time \\
\hline \multirow[t]{5}{*}{ rw100 } & 10 & 5301 & RES04/PBS & 530 & 0.000 & $\overline{0.02}$ \\
\hline & 20 & 277 & RES04/PBS & 277 & 0.000 & $<\epsilon$ \\
\hline & 30 & 213 & RES04/PBS & 213 & 0.000 & $<\epsilon$ \\
\hline & 40 & 187 & RES04/PBS & 187 & 0.000 & $<\epsilon$ \\
\hline & 50 & 172 & RES04/PBS & 172 & 0.000 & $<\epsilon$ \\
\hline \multirow[t]{6}{*}{ rw250 } & 10 & 3691 & RES04/PBS & 3691 & 0.000 & 0.15 \\
\hline & 25 & 1360 & PBS & 1364 & 0.262 & 0.11 \\
\hline & 50 & 713 & RES04/PBS & 713 & 0.000 & 0.49 \\
\hline & 75 & 523 & RES04/PBS & 523 & 0.000 & 0.20 \\
\hline & 100 & 444 & RES04/PBS & 444 & 0.000 & 0.01 \\
\hline & 125 & 411 & RES04/PBS & 411 & 0.000 & 0.01 \\
\hline \multirow[t]{8}{*}{ rw500 } & 10 & 16108 & RES04/PBS & 16108 & 0.000 & 4.48 \\
\hline & 25 & 5626 & PBS & 5681 & 0.978 & 13.46 \\
\hline & 50 & 2626 & PBS & 2628 & 0.068 & 8.09 \\
\hline & 75 & 1757 & RES04/PBS & 1757 & 0.000 & 3.10 \\
\hline & 100 & 1379 & PBS & 1380 & 0.073 & 5.17 \\
\hline & 150 & 1024 & RES04/PBS & 1024 & 0.000 & 22.04 \\
\hline & 200 & 893 & RES04/PBS & 893 & 0.000 & 0.04 \\
\hline & 250 & 833 & RES04/PBS & 833 & 0.000 & 0.01 \\
\hline \multirow[t]{9}{*}{ rw1000 } & 10 & 67811 & RES04/PBS & 67811 & 0.000 & 7.43 \\
\hline & 25 & 24896 & RES04/PBS & 24896 & 0.000 & 14.62 \\
\hline & 50 & 11259 & PBS & 11296 & 0.322 & 37.58 \\
\hline & 75 & 7134 & PBS & 7168 & 0.477 & 101.55 \\
\hline & 100 & 5210 & PBS & 5225 & 0.302 & 51.97 \\
\hline & 200 & 2704 & PBS & 2710 & 0.221 & 33.89 \\
\hline & 300 & 2018 & RES04/PBS & 2018 & 0.000 & 38.29 \\
\hline & 400 & 1734 & RES04/PBS & 1734 & 0.000 & 0.51 \\
\hline & 500 & 1614 & RES04/PBS & 1614 & 0.000 & 0.09 \\
\hline
\end{tabular}

PBS-1 RESULTS, FROM 9 INDEPENDENT TRIALS, FOR THE CLASS RW [16]: BEST KNOWN COST (BEST); SOURCE OF BEST KNOWN COST (SOURCE) IS SHOWN AS RES04 [16] OR PBS (THIS STUDY); MEDIAN VALUES (MED), AVERAGE PERCENTAGE COST ERROR (\%ERR) AND AVERAGE CPU TIMES IN SECONDS (TIME). AVERAGE CPU TIMES

$$
<0.01 \text { ARE SHOWN AS ' }<\epsilon \text { '. }
$$

computational efficiency of the algorithms and the quality of the results produced. The quality of the PBS results has been documented in terms of the median $p$-median cost and average percentage error from the best known cost over 9 or 100 trials. With regard to these quality of results metrics, it must be noted that seemingly small differences in the median $p$-median costs and average percentage cost error may in fact represent major differences in algorithm performance, since (as in many hard combinatorial optimisation problems) finding $p$-median solutions with slightly higher costs is typically substantially easier than finding those with minimal costs.

The computational efficiency of PBS has been documented in terms of the CPU time used on a computer processor that

\begin{tabular}{|c|c|c|c|c|c|c|c|}
\hline \multirow[b]{2}{*}{$\mathrm{p}$} & \multicolumn{2}{|c|}{ Best Known } & \multicolumn{2}{|c|}{ Average } & \multicolumn{3}{|c|}{ PBS-10 PBS-20 PBS-30 } \\
\hline & Best & Source & MED & \%ERR & Time & Time & Time \\
\hline 10 & 101249.47 & HMP01/PBS & 101249.55 & 0.000 & $<\epsilon$ & $<\epsilon$ & 0.11 \\
\hline 20 & 57857.55 & HMP01/PBS & 57857.94 & 1 & 0.44 & $<\epsilon$ & 0. \\
\hline 30 & & CRA0 & 013.48 & 0. & 4.22 & 1.56 & \\
\hline 40 & 35002.02 & CRA0 & 5002.52 & 0.002 & 0.44 & $<\epsilon$ & $<$ \\
\hline 50 & 29089 & CRA & 29090.23 & 0.002 & 0.78 & 0.67 & 0.33 \\
\hline 60 & 2516 & $\mathrm{CR}$ & 12 & 0.003 & 10.22 & 5.00 & 3.78 \\
\hline 70 & 22125.46 & CRAC & 22126.03 & 0.003 & 4.67 & 2.22 & 1.67 \\
\hline 80 & 19870.28 & CRA & 19870.85 & 0.003 & 8.67 & 4.56 & 3.85 \\
\hline 90 & 179 & $\mathrm{CR}$ & & & 56 & 2.56 & 2.22 \\
\hline 100 & 1655 & HMP9 & 2.22 & 0.006 & 10.22 & 9.33 & 4.89 \\
\hline 150 & 12026.41 & $\mathrm{PE}$ & 12034.98 & 0.071 & 16.56 & 8.89 & 7.67 \\
\hline 200 & 9 & & 0.18 & 0.038 & 17.67 & 11.22 & 8.78 \\
\hline 250 & & & & 0.1 & 10.67 & 7.11 & 5.89 \\
\hline 300 & 66 & & 79 & 0.099 & 32.89 & 17.11 & 14.44 \\
\hline 350 & 5719.30 & $\mathrm{PE}$ & 726.81 & 0.131 & 28.56 & 17.11 & 14.56 \\
\hline 400 & 5006.75 & $\mathrm{~PB}$ & 5010.11 & 0.067 & 249.22 & 82.89 & 51.00 \\
\hline 450 & & & 4478.36 & 0.112 & 193.67 & 131.78 & 85.44 \\
\hline 500 & 4046.86 & $\mathrm{~PB}$ & 4049.41 & 0.063 & 79.11 & 61.78 & 47.1 \\
\hline
\end{tabular}

PBS-10, PBS-20 AND PBS-30 RESULTS, FROM 9 INDEPENDENT TRIALS, FOR THE CLASS $f 1400$ [21]: BEST KNOWN COST (BEST); SOURCE OF BEST KNOWN COST (SOURCE) IS SHOWN AS HMP01 [9], CRA04 [11], HMP97 [10] OR PBS (THIS STUDY); AVERAGE MEDIAN COST OVER ALL TRIALS (MED); AVERAGE PERCENTAGE COST ERROR OVER ALL TRIALS (\%ERR); AND AVERAGE ELAPSED TIMES IN SECONDS

(Time). AVERAgE ElAPSED times $<0.01$ ARE SHOWN AS ' $<\epsilon$ '.

\begin{tabular}{|c|c|c|c|c|c|c|c|}
\hline \multirow[b]{2}{*}{$\mathrm{p}$} & \multicolumn{2}{|c|}{ Best Known } & \multicolumn{2}{|c|}{ Average } & \multirow{2}{*}{$\begin{array}{c}\text { PBS-10 } \\
\text { Time }\end{array}$} & \multirow{2}{*}{$\begin{array}{c}\text { PBS-20 } \\
\text { Time }\end{array}$} & \multirow{2}{*}{$\begin{array}{c}\text { PBS-30 } \\
\text { Time }\end{array}$} \\
\hline & Best & Source & MED & \%ERR & & & \\
\hline$\overline{10}$ & 213082.03 & $\overline{R E S 04 / P B S}$ & 1213082.03 & 0.000 & 0.33 & 0.33 & 0.11 \\
\hline 20 & 840844.53 & RES04/PBS & 840844.53 & 0.000 & 5.00 & 5.44 & 4.11 \\
\hline 30 & 677272.22 & PBS & 677404.52 & 0.020 & 549.00 & 151.11 & 128.78 \\
\hline 40 & 571887.75 & RES04/PBS & 571887.75 & 0.000 & 15.44 & 9.78 & 11.11 \\
\hline 50 & 507558.16 & CRA04/PBS & 507640.13 & 0.016 & 70.11 & 16.22 & 11.33 \\
\hline 60 & 460771.87 & RES04/PBS & 460794.48 & 0.005 & 30.00 & 23.22 & 22.44 \\
\hline 70 & 426068.24 & RES04/PBS & 426140.81 & 0.017 & 41.11 & 24.44 & 21.33 \\
\hline 80 & 397491.10 & PBS & 397572.43 & 0.020 & 39.22 & 24.00 & 22.11 \\
\hline 90 & 373241.86 & PBS & 373476.07 & 0.063 & 35.44 & 25.11 & 18.33 \\
\hline 100 & 352612.79 & PBS & 352737.86 & 0.035 & 35.67 & 33.11 & 28.56 \\
\hline 150 & 281181.68 & PBS & 281309.07 & 0.045 & 86.22 & 43.67 & 31.22 \\
\hline 200 & 238353.32 & PBS & 238423.75 & 0.030 & 259.22 & 80.78 & 81.22 \\
\hline 250 & 209218.48 & PBS & 209323.19 & 0.050 & 95.11 & 80.00 & 60.22 \\
\hline 300 & 187691.38 & PBS & 187759.60 & 0.036 & 153.44 & 88.22 & 78.11 \\
\hline 350 & 170942.24 & PBS & 171045.07 & 0.060 & 121.44 & 96.22 & 74.00 \\
\hline 400 & 157029.10 & PBS & 157071.22 & 0.027 & 298.11 & 190.89 & 132.22 \\
\hline 450 & 145375.40 & PBS & 145417.03 & 0.029 & 422.89 & 252.11 & 175.33 \\
\hline 500 & 135466.28 & PBS & 135506.75 & 0.030 & 1093.00 & 437.33 & 238.44 \\
\hline 550 & 126838.11 & PBS & 126895.63 & 0.045 & 1449.11 & 749.56 & 383.22 \\
\hline 600 & 119089.89 & PBS & 119133.75 & 0.037 & 1113.33 & 504.67 & 318.67 \\
\hline 650 & 112029.04 & PBS & 112074.34 & 0.040 & 1518.56 & 638.11 & 497.56 \\
\hline 700 & 105844.60 & PBS & 105888.23 & 0.041 & 821.33 & 515.89 & 380.00 \\
\hline 750 & 100353.68 & PBS & 100390.90 & 0.037 & 1307.44 & 678.78 & 516.33 \\
\hline 800 & 95405.89 & PBS & 95431.64 & 0.027 & 1325.00 & 785.22 & 532.44 \\
\hline 850 & 91000.61 & PBS & 91032.43 & 0.035 & 1369.78 & 690.56 & 571.33 \\
\hline 900 & 86973.11 & PBS & 87021.68 & 0.056 & 1619.56 & 1050.33 & 553.56 \\
\hline 950 & 83275.69 & PBS & 83298.62 & 0.028 & 1830.56 & 956.22 & 606.33 \\
\hline 1000 & 79853.82 & PBS & 79869.25 & 0.019 & 1633.67 & 832.67 & 565.00 \\
\hline
\end{tabular}

PBS-10, PBS-20 AND PBS-30 RESULTS, FROM 9 INDEPENDENT TRIALS, FOR THE CLASS pcb3038 [21]: BEST KNOWN COST (BEST); SOURCE OF BEST KNOWN COST (SOURCE) IS SHOWN AS RES04 [16], CRA04 [11] OR PBS (THIS STUDY); MEDIAN COST (MED); AVERAGE PERCENTAGE COST ERROR (\%ERR); AND AVERAGE ELAPSED TIMES IN SECONDS (TIME).

has been benchmarked using a publicly available benchmark program. However, in previous studies, computing hardware has basically been documented in terms of CPU type (and, sometimes, CPU clock rate) which only allows a very basic means of comparison (i.e., by scaling based on the 


\begin{tabular}{|c|c|c|c|c|c|c|c|}
\hline \multirow[b]{2}{*}{$\mathrm{p}$} & \multicolumn{2}{|c|}{ Best Known } & \multicolumn{2}{|c|}{ Average } & PBS-10 & $\overline{\text { PBS-20 }}$ & \multirow{2}{*}{$\begin{array}{c}\text { PBS-30 } \\
\text { Time }\end{array}$} \\
\hline & Best & Source & MED & \%ERR & Time & Time & \\
\hline 10 & 9794951.00 & HMP01 & 9794973.65 & 0.000 & 24.78 & 6.78 & 11.67 \\
\hline 20 & 6718848.19 & RES04/PBS & 6719017.83 & 0.003 & 98.56 & 43.11 & 35.89 \\
\hline 30 & 5374936.14 & RES04/PBS & 5375923.84 & 0.018 & 76.56 & 60.11 & 38.22 \\
\hline 40 & 4550327.09 & PBS & 4550499.03 & 0.004 & 70.67 & 47.33 & 37.00 \\
\hline 50 & 4032379.97 & RES04/PBS & 4032621.78 & 0.006 & 85.44 & 40.00 & 52.11 \\
\hline 60 & 3642064.70 & PBS & 3642870.18 & 0.022 & 95.89 & 71.44 & 51.67 \\
\hline 70 & 3343617.76 & PBS & 3344853.43 & 0.037 & 112.78 & 97.56 & 72.22 \\
\hline 80 & 3094507.17 & PBS & 3095359.40 & 0.028 & 139.22 & 97.11 & 80.22 \\
\hline 90 & 2893234.39 & PBS & 2894843.70 & 0.056 & 733.78 & 414.67 & 81.78 \\
\hline 100 & 2725020.51 & PBS & 2725500.78 & 0.018 & 171.67 & 118.11 & 117.78 \\
\hline 150 & 2147817.00 & PBS & 2148721.38 & 0.042 & 166.11 & 113.56 & 122.22 \\
\hline 200 & 1808010.37 & PBS & 1808643.12 & 0.035 & 273.22 & 196.56 & 203.00 \\
\hline 250 & 1569830.31 & PBS & 1570412.75 & 0.037 & 293.11 & 226.33 & 203.89 \\
\hline 300 & 1394045.57 & PBS & 1394341.18 & 0.021 & 378.44 & 332.56 & 284.33 \\
\hline 350 & 1256775.39 & PBS & 1257084.61 & 0.025 & 606.00 & 448.89 & 377.56 \\
\hline 400 & 1145631.85 & PBS & 1145942.24 & 0.027 & 467.11 & 371.56 & 314.33 \\
\hline 450 & 1053303.41 & PBS & 1053717.23 & 0.039 & 638.11 & 328.89 & 311.67 \\
\hline 500 & 973982.54 & PBS & 974230.36 & 0.025 & 765.89 & 508.56 & 403.44 \\
\hline 600 & 848266.43 & PBS & 848492.37 & 0.027 & 721.00 & 458.11 & 413.89 \\
\hline 700 & 752047.96 & PBS & 752259.40 & 0.028 & 674.33 & 523.67 & 446.56 \\
\hline 800 & 676778.00 & PBS & 676952.90 & 0.026 & 1396.56 & 820.00 & 655.00 \\
\hline 900 & 613352.32 & PBS & 613494.23 & 0.023 & 444.78 & 1111.89 & 741.44 \\
\hline 1000 & 558801.23 & PBS & 558941.68 & 0.025 & 1770.22 & 909.56 & 761.22 \\
\hline 1100 & 511804.96 & PBS & 511923.31 & 0.023 & 1892.89 & 641.78 & 571.22 \\
\hline 1200 & 470290.06 & PBS & 470408.35 & 0.025 & 2392.11 & 894.44 & 702.00 \\
\hline 1300 & 433587.06 & PBS & 433675.29 & 0.020 & 1573.11 & 1072.33 & 813.44 \\
\hline 1400 & 401829.22 & PBS & 401931.31 & 0.025 & 3778.00 & 1403.00 & 250.44 \\
\hline 1500 & 374001.06 & PBS & 374051.86 & 0.014 & 6409.11 & 3252.44 & 2419.00 \\
\hline
\end{tabular}

PBS-10, PBS-20 AND PBS-30 RESULTS, FROM 9 INDEPENDENT TRIALS, FOR THE CLASS rl5934 [21]: BEST KNOWN COST (BEST); SOURCE OF BEST KNOWN COST (SOURCE) IS SHOWN AS HMP01 [9], RES04 [16] OR PBS (THIS STUDY); MEDIAN COST (MED); AVERAGE PERCENTAGE COST ERROR (\%ERR); AND AVERAGE ELAPSED TIMES IN SECONDS (TIME).

\begin{tabular}{rccccc}
\hline & \multicolumn{3}{c}{ Best Known } & \multicolumn{3}{c}{ Previous Best Known } & \\
\cline { 2 - 3 } \cline { 5 - 6 } Best & Source & Best & Source & \%IMP \\
\hline 1005850643.77 & PBS & 5855395.00 & CRA04 & 0.081 \\
200 & 4003810.27 & PBS & 4017110.50 & CRA04 & 0.332 \\
300 & 3194524.90 & PBS & 3210784.00 & CRA04 & 0.509 \\
400 & 2696220.83 & PBS & 2712334.50 & CRA04 & 0.598 \\
500 & 2357272.95 & PBS & 2367523.00 & CRA04 & 0.435 \\
6002112972.51 & PBS & 2125355.50 & CRA04 & 0.586 \\
7001923107.93 & PBS & 1932731.75 & CRA04 & 0.500 \\
8001766617.96 & PBS & 1775417.62 & CRA04 & 0.498 \\
9001636303.31 & PBS & 1644025.75 & CRA04 & 0.472 \\
10001523174.03 & PBS & 1531481.88 & CRA04 & 0.545 \\
\hline \multicolumn{5}{c}{ TABLE VIII }
\end{tabular}

PBS RESULTS FOR THE CLASS $r$ l11849 [21]: BEST KNOWN COST (BEST); SOURCE OF BEST KNOWN COST (SOURCE) IS SHOWN AS PBS (THIS STUDY); PREVIOUS BEST KNOWN COST (BEST); SOURCE OF PREVIOUS BEST KNOWN COST (SOURCE) IS SHOWN AS CRA04 [11]; AND AVERAGE PERCENTAGE COST IMPROVEMENT FOR THE PBS RESULT (\% IMP).

computer CPU clock speed which, for example, takes no account of other features, such as memory caching, memory size, hardware architecture, etc.). In addition, differences in experimental protocol can have a major effect on what is actually included in published CPU times for hueristics. For PBS, VNS [10] and VNDS [9], the average of the CPU / elapsed times until the target $p$-median cost was found (or, if not found, the CPU / elapsed time at which the lowest $p$-median cost solution was located) for each instance are documented. However, for HYBRID [16], CGLS [22], LSH [13] and ADE [15], the reported CPU times are obtained by running the algorithm for some fixed computational limit (e.g. for HYBRID, 32 multistart iterations). It is not reported if these experiments terminated early for instances where a known optimal solution was located.

One method of comparing PBS with other algorithms is to build on the comparisons performed in [16] by documenting the average percentage cost error for each benchmark class and PBS-1 CPU times as ratios of the average percentage cost error and CPU times reported in [16]. Table IX shows the results of this extrapolation. With regard to Table IX, for consistency, the average percentage cost errors for PBS-1 are related to the best known costs published in [16]. Clearly, in terms of robustness and quality of results, PBS-1 achieves excellent results. With regard to computational requirements, due to differences in computers and experimental protocols, the CPU comparisons in Table IX is impossible to analyse with any degree of accuracy.

\begin{tabular}{|c|c|c|c|c|}
\hline \multirow{2}{*}{\multicolumn{5}{|c|}{$\begin{array}{c} \\
\text { Class }\end{array}$}} \\
\hline & & & & \\
\hline$\overline{\text { ORLIB }}$ & 0.000 & 0.1010 .000 & 0.116 & $\overline{0.007}$ \\
\hline SL & 0.000 & 0.6910 .332 & -- & -- \\
\hline GR & 0.000 & --0.727 & -- & -- \\
\hline & \multicolumn{4}{|c|}{ CPU Ratio } \\
\hline \multicolumn{5}{|c|}{ YBRID CGLS LS } \\
\hline$\overline{\text { ORLIB }}$ & 1.00 & 55.984 .13 & 0.46 & 5.47 \\
\hline SL & 1.00 & $0.51 \quad 24.20$ & -- & -- \\
\hline GR & 1.00 & $--\quad 1.11$ & -- & -- \\
\hline
\end{tabular}

AVERAGE PERCENTAGE COST ERRORS (\%ERR) AND RATIOS OF REPORTED AVERAGE CPU TIMES (ADAPTED FROM [16]) FOR PBS-1, HYBRID [16], CGLS [22], LSH [13], VNDS [9], VNS [10] FOR THE ORLIB, SL AND GR CLASSES. ENTRIES IN ITALICS SIGNIFY THAT NOT ALL INSTANCES OF A CLASS WERE INCLUDED WHILE - - INDICATES NO INSTANCES OF THAT CLASS WERE TESTED.

\begin{tabular}{lcccccc}
\hline Class & PBS-1 & HYBRID CGLS LSH & VNDS VNS \\
\hline ORLIB & 0.03 & 1.00 & 55.98 & 4.13 & 0.46 & 5.47 \\
SL & 0.06 & 1.00 & 0.51 & 24.20 & -- & -- \\
GR & 0.05 & 1.00 & -- & 1.11 & -- & -- \\
\hline \multicolumn{7}{c}{ TABLE X }
\end{tabular}

RATIOS OF REPORTED AVERAGE CPU TIMES (ADAPTED FROM [16]) FOR PBS-1, HYBRID [16], CGLS [22], LSH [13], VNDS [9], VNS [10].

ENTRIES IN ITALICS SIGNIFY THAT NOT ALL INSTANCES OF A CLASS WERE INCLUDED WHILE - - INDICATES NO INSTANCES OF THAT CLASS WERE TESTED.

A similar comparison of PBS-1 with ADE [15] on the instances of the ORLIB class gives, for PBS-1, an average percentage cost error of 0.000 and an average CPU time of 0.48 seconds while the corresponding figures for ADE are 0.110 and 18.395 seconds respectively. This represents a PBS-1 to ADE ratio of 0.00 for average percentage cost error and 0.03 for average CPU times (note that this comparison also ignores differences in computing hardware and experimental protocol).

Fundamentally, comparing PBS with other algorithms is difficult of differences in experimental protocols, differences in computer processor speeds amd also because the quality of results (as measured by median cost and average percentage 
cost error over the trials), and computational requirements (measured by average CPU time for single processor algorithms and / or average elapsed time for multi-processor algorithms) are related by a non-linear relationship. This complex situation can, to a degree, be simplified by performing PBS- 1 trials in which the target $p$-median cost is set so that the resulting median cost and / or average percentage cost error are as close as possible to those obtained in previous studies. This reduces the impact of the differences in the quality of results on the comparisons and the computer processor and experimental differences become the major issues when comparing results obtained in this study with those obtained in previous studies. Table XI shows the results obtained where, for all the rw1000 instances, PBS-1 was run over 9 trials with a target cost that produced median $p$ median costs that are close to those reported in [16]. The average overall ratio of the CPU times required by PBS-1 to those reported in [16] is 0.09 (as compared to an average of 0.05 from Table X). Table XII provides a comparison

\begin{tabular}{|c|c|c|c|c|c|c|}
\hline \multirow[b]{2}{*}{$\mathrm{p}$} & \multicolumn{3}{|c|}{ PBS-1 } & \multicolumn{3}{|c|}{ HYBRID } \\
\hline & MED & $\%$ ERR & Time & MED & \%ERR & Time \\
\hline 10 & 68136 & 0.437 & 1.16 & 68136 & 0.466 & 256.3 \\
\hline 25 & 24923 & 0.131 & 4.68 & 24964 & 0.451 & 293.5 \\
\hline 50 & 11360 & 0.454 & 4.17 & 11360 & 0.602 & 169.1 \\
\hline 75 & 7203 & 0.528 & 18.80 & 7207 & 0.576 & 160.1 \\
\hline 100 & 5255 & 0.574 & 10.56 & 5259 & 0.598 & 109.8 \\
\hline 200 & 2710 & 0.144 & 33.79 & 2710 & 0.164 & 100.4 \\
\hline 300 & 2018 & 0.000 & 35.98 & 2018 & 0.022 & 71.5 \\
\hline 400 & 1734 & 0.000 & 0.56 & 1734 & 0.000 & 73.5 \\
\hline 500 & 1614 & 0.000 & 0.10 & 1614 & 0.000 & 55.9 \\
\hline
\end{tabular}

AVERAGE CPU RESUlTS FOR PBS-1, OVER 9 TRIALS, ON THE RW 1000 INSTANCES WITH A PBS- 1 TARGET COST THAT PRODUCED MEDIAN $p$-MEDIAN COSTS CLOSE TO THOSE REPORTED FOR HYBRID [16]: MEDIAN COST (MED); AVERAGE PERCENTAGE COST ERROR (\%ERR); AND REPORTED AVERAGE CPU TIME (TIME).

between PBS and HYBRID on instances from TSPLIB. These TSPLIB instances where selected on the basis that the best known cost used in [16] and this study were identical and the average percentage cost errors comparable. The ratio of the average PBS CPU time to the average HYBRID CPU time for these instances is 0.12 . It should be noted that PBS and HYBRID behave in rather different ways on the TSPLIB instances as $p$ increases. For PBS, the average CPU time, in general, increases as $p$ increases while, for HYBRID, the average CPU time starts at a large value, decreases as $p$ approaches 70 (fl1400) / 150 (pcb3038) / 300 (rl5934) and then begins to increase. While these comparisons with other studies suggest that PBS has excellent CPU performance, no final conclusive statement can be made as to the relative CPU performance of the algorithms compared in this section, as differences in computer processor speed and differences as to what is actually included in the time measurements still prevent any concrete conclusions being drawn. The more complete documentation of computer processor performance presented in this study should allow future researchers to make more meaningful comparisons.

\begin{tabular}{|c|c|c|c|c|c|c|c|}
\hline \multicolumn{2}{|c|}{ Instance } & \multicolumn{3}{|c|}{ PBS-1 } & \multicolumn{3}{|c|}{ HYBRID } \\
\hline Name & $\mathrm{p}$ & MED & \%ERR & Time & MED & \%ERR & Time \\
\hline fl1400 & 10 & 101249.55 & 0.000 & 0.10 & 101249.55 & 0.000 & 118.5 \\
\hline \multirow{4}{*}{ pcb3038 } & 10 & 1213082.03 & 0.000 & 3.30 & 1213082.03 & 0.000 & 1806.3 \\
\hline & 20 & 840844.53 & 0.000 & 50.00 & 840844.53 & 0.003 & 943.4 \\
\hline & 40 & 571887.75 & 0.000 & 154.40 & 571887.75 & 0.004 & 492.6 \\
\hline & 70 & 426140.81 & 0.017 & 411.10 & 426153.31 & 0.020 & 470.9 \\
\hline \multirow[t]{3}{*}{ rl5934 } & 10 & 9794973.65 & 0.000 & 247.80 & 9794973.65 & 0.000 & 8687.1 \\
\hline & 20 & 6719017.83 & 0.003 & 985.60 & 6719026.03 & 0.003 & 4779.6 \\
\hline & 30 & 5375923.84 & 0.018 & 765.60 & 5376040.45 & 0.017 & 4515.1 \\
\hline
\end{tabular}

TABLE XII

MEdian Cost (MED) AND REPorted AVERAge CPU TIME (TIME)

RESULTS FOR PBS-1 AND HYBRID [16] FOR THOSE TSPLIB

INSTANCES WHERE THE BEST KNOWN COST WAS IDENTICAL AND THE AVERAGE PERCENTAGE COST ERROR (\%ERR) COMPARABLE. THE CPU TIMES FOR PBS-1 WERE OBTAINED BY SCALING THE EQUIVALENT PBS-10 TIMES BY A FACTOR OF 10.

\section{DISCUSSION}

To gain a deeper understanding of the run-time behaviour of PBS and the efficacy of its underlying mechanisms, additional empirical analyses were performed. Specifically, the variability in run-time between multiple independent runs of PBS on the same problem instance; the effect of the population size and the effect of adding additional computer processors; and the efficiency of the genetic operators were investigated. These investigations were performed using two instances, $r w 1000$ with $p=50$ and $f 1400$ with $p=150$ selected because, firstly, they are of reasonable size and difficulty and, secondly, rw1000 is non-symmetric and is specified by a distance matrix while $f 1400$ is symmetric and is specified using the $(\mathrm{x}, \mathrm{y})$ coordinates of each vertex.

\section{A. Variability in Run-Time}

The variability of run-time between multiple independent runs on a given problem is an important aspect of the behaviour of stochastic algorithms such as PBS. Following the methodology of [23], this aspect is presented based on run-time distributions (RTDs) of PBS-1 on the two reference instances.

As can be seen from the empirical RTD graphs shown in Figure 1 (based on 100 independent trials that all reached the target $p$-median cost), PBS- 1 shows a reasonably large variability in run-time with that for instance rw1000 being larger than that of instance fl1400 (a possible reason for this may be that fewer genetic crossover operators are available for rw1000). This observation of a reasonably large variability in run-time is consistent with similar results for other high-performance stochastic search algorithms, e.g., for maximum clique [24] and scheduling problems [25]. Similar observations were made for PBS for most of the other PM benchmark instances.

\section{B. Population Size}

The effect of the PBS-1 population size $(|P|)$ on the average CPU time when optimising instance $f 1400, p=150$, target $p$-median cost 12035.12 , is shown in Figure 2. It would appear that, when $|P|$ is very small the genetic operators are unable to generate sufficient diversity in starting points 

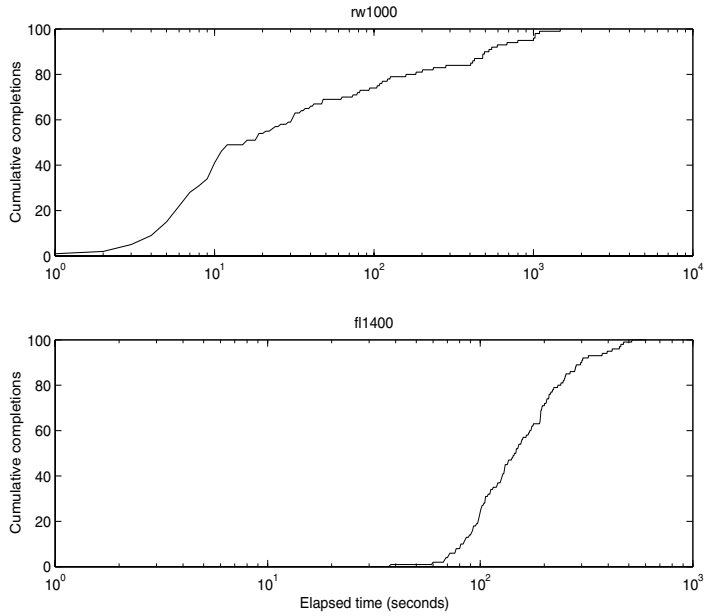

Fig. 1. Run Time Distributions (RTDs) of elapsed time for PBS-1 for 100 trials on the rw1000, $p=50$, target $p$-median cost $=11296$ and fl1400, $p$ $=150$ instances, target $p$-median cost $=12035.12$.

for the HLS to allow the search to progress quickly and, when $|P|$ is large, computational effort is basically wasted on applying the genetic operators and HLSs to $p$-median solutions which are distant from the target $p$-median solution and give a very small probability of locating better solutions.

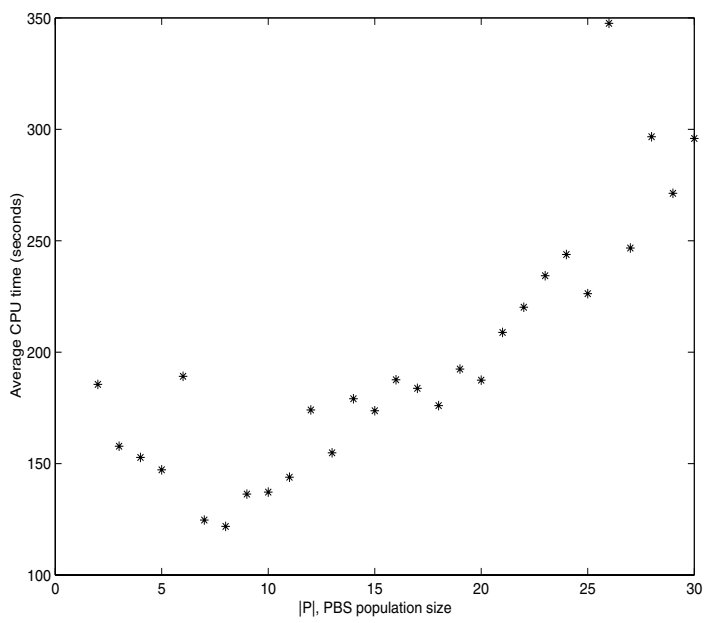

Fig. 2. PBS-1 average CPU time, averaged over 100 successful trials of instance fl1400, $p=150$, as the population size varies in the range $2, \ldots, 30$. The target $p$-median cost was 12035.12 .

\section{Computer Processors}

The effect of adding computer processors for PBS to utilise is shown in Figure 3 for the instance rw1000 with $p=50$, target $p$-median cost $=5128$ with maximum generations set to 200. As would be expected, there is an initial rapid decrease in average elapsed time with a subsequent levelling off as the number of processors increases above four. Also shown is the predicted elapsed time with $i$ processors, $t_{i}$, calculated by $t_{i}=t_{1} / i$.

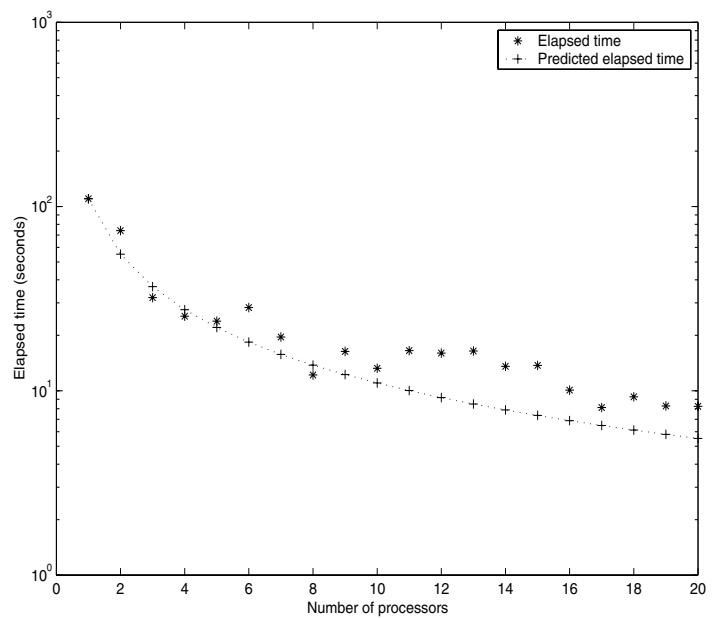

Fig. 3. Effect of the number of processors on the PBS elapsed time for the instance rw 1000 with $p=50$, target $p$-median cost $=5128$ with a maximum generations of 200. Also shown is the predicted elapsed time with $i$ processors, $t_{i}$, calculated by $t_{i}=t_{1} / i$.

\section{Genetic Operators}

The percentage of times that individual crossover and mutation operators were responsible for locating the target $p$-median cost, from 9 trials on all 74 instances of the $f 1400$, pcb3038 and rl5934 classes was for $\mathcal{X}_{1}, 8.52 \%$, for $\mathcal{X}_{2}$ $14.38 \%$, for $\mathcal{X}_{3} 30.76 \%$, for $\mathcal{X}_{4} 14.11 \%$, for $\mathcal{X}_{5} 11.32 \%$, for $\mathcal{X}_{6} 9.05 \%$, for $\mathcal{X}_{7} 5.73 \%$ and for the mutation operator $6.13 \%$. While there is a reasonable range in these figures, given that no genetic operator achieved a zero success rate, all remain in the final version of PBS.

Figure 4 gives more detailed information on the overall performance of the genetic operators in that it shows the evolution, using instance $f 1400, p=150$, for each application of a genetic operator and subsequent HLS, of the average $p$ median cost for the parents to the actual $p$-median cost of the child solutions after the genetic operator (which becomes the input to the HLS) and then the final $p$-median cost as a result of the HLS. The effectiveness of the genetic operators in generating a wide range of starting points, from a population of eight solutions, for the HLS is clearly demonstrated. In addition, it was observed that the phenotype genetic operators appeared to become more effective as $p$ increased.

\section{CONCLUSions AND Future Work}

This study has demonstrated that by applying the general paradigm of using a hybrid local search, within a population based meta-heuristic, to the $p$-median problem, the state of the art in PM solving can be improved. In summary, with regard to the robustness and quality of the PBS results, for the 171 benchmark instances, PBS found 72 new best known results, located 88 of the currently best known / optimal 

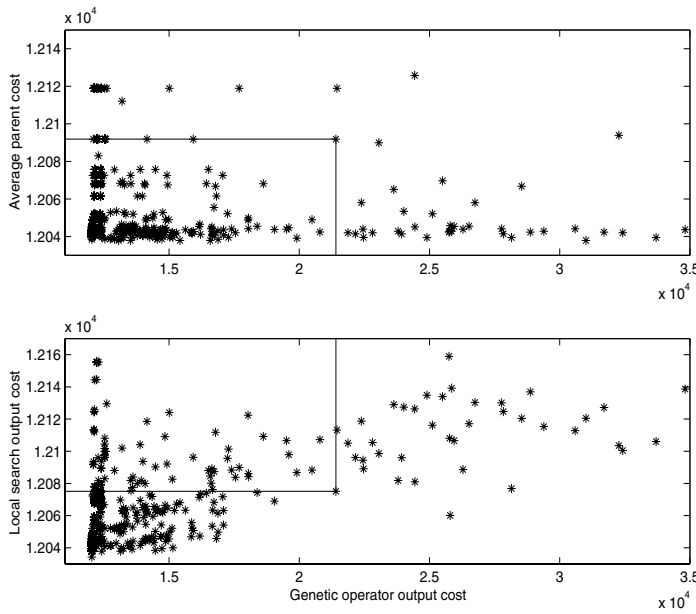

Fig. 4. The effectiveness of the genetic operators and HLS demonstrated by tracing the evolution of the $p$-median cost from average parent cost, child cost and finally the cost after the application of the HLS for instance $f 1400$, $p=150$. The line traces the changes in $p$-median cost for a particular application of a genetic operator / HLS sequence.

results and failed to obtain the best known results for the remaining 11 instances (average percentage cost error for the failed instances is 0.002). With regard to computational performance, while the comparisons performed with other studies suggest that PBS has excellent performance, due to difficulties in realistically comparing computational performance with that documented for other algorithms, no final conclusive statement can be made as to the relative CPU performance of PBS as compared to other algorithms.

Given the performance of PBS on the standard PM instances reported here, the algorithm clearly has substantial potential to provide the basis for high-performance algorithms for other clustering combinatorial optimisation problems such as capacitated pmedian, maximum covering, uncapacitated / capacitated facility location and set covering.

\section{REFERENCES}

[1] C. ReVelle and R. Swain, "Central facilities location," Geographical Analysis, vol. 2, pp. 30-42, 1970.

[2] B. C. Tansel, R. L. Francis, and T. J. Lowe, "Location on networks: a survey," Management Science, vol. 29, pp. 482-511, 1983.

[3] M. R. Rao, "Cluster analysis and mathematical programming," Journal of the American Statistical Association, vol. 66, pp. 622-626, 1971.

[4] O. Kariv and L. Hakimi, "An algorithmic approach to nework location problems, part ii the p-medians," SIAM Journal of Applied Mathematics, vol. 37, pp. 539-560, 1979.

[5] J. E. Beasley, "A note on solving large p-median problems," European Journal of Operational Research, vol. 21, pp. 270-273, 1985.

[6] R. D. Galvão, "A dual-bounded algorithm for the p-median problem," Operations Research, vol. 28, pp. 1112-1121, 1980.

[7] K. E. Rosing, C. S. ReVelle, and H. Rosing-Vogelaar, "The p-median and its linear programming relaxation: An approach to large problems," Journal of the Operational Research Society, vol. 30, pp. 815-823, 1979.

[8] R. M. Nauss and R. E. Markland, "Theory and application of an optimizing procedure for lock box location analysis," Management Science, vol. 27, pp. 855-865, 1981.
[9] P. Hansen, N. Mladenović, and D. Perez-Brito, "Variable neighborhood decomposition search," Journal of Heuristics, vol. 7, pp. 335-350, 2001.

[10] P. Hansen and N. Mladenović, "Variable neighborhood search for the p-median," Location Science, vol. 5, pp. 207-226, 1997.

[11] T. Cranic, M. Gendreau, P. Hansen, and N. Mladenović, "Cooperative parallel variable neighbourhood search for the $p$-median," Journal of Heuristics, vol. 10, pp. 293-314, 2004.

[12] E. D. Taillard, "Heuristic methods for large centroid clustering problems," Journal of Heuristics, vol. 9, pp. 51-74, 2003.

[13] E. L. F. Senne and L. A. N. Lorena, "Langrangean/surrogate heuristics for p-median problems," in Computing Tools for Modeling, Optimization and Simulation Interfaces in Computer Science and Operations Research, M. Laguna and J. L. Gonzalez-Velarde, Eds. Kluwer, 2000, pp. $115-130$.

[14] — "Stabilizing column generation using lagrangean/surrogate relaxation an application to p-median location problems," European Journal of Operational Research, 2002.

[15] O. Alp, E. Erkut, and Z. Drezner, "An efficient genetic algorithm for the $p$-median problem," Annals of Operations Research, vol. 122, pp. 21-42, 2003.

[16] M. Resende and R. Werneck, "A hybrid heuristic for the $p$-median problem," Journal of Heuristics, vol. 10, pp. 59-88, 2004.

[17] A. Kuehn and M. Hamburger, "A heuristic program for locating warehouses," Management Science, vol. 9, pp. 643 - 666, 1963.

[18] S. Kirkpatrick, J. Gellatt, and P. Vecchi, "Optimization by simulated annealing," Science, vol. 220, pp. 671 - 680, 1983.

[19] F. Glover, "Tabu search - part 1," ORSA Journal on Computing, vol. 1, pp. 190 - 206, 1989.

[20] R. D. Galvão and C. S. ReVelle, "A lagrangean heuristic for the maximal covering problem," European Journal of Operational Research, vol. 18, pp. 114-123, 1996.

[21] G. Reinelt, "Tsplib a traveling salesman problem library," ORSA Journal on Computing, vol. 3, pp. 376-384, 1991.

[22] E. L. F. Senne, "Personal communication to m.c.g. resende," 2002.

[23] H. H. Hoos and T. Stützle, Stochastic Local Search: Foundations and Applications. Morgan Kaufmann Publishers, USA, 2004.

[24] W. Pullan and H. Hoos, "Dynamic local search for the maximum clique problem," Journal of Artificial Intelligence Research, vol. 26, pp. $159-185,2006$.

[25] J. Watson, L. Whitley, and A. Howe, "Linking search space structure, run-time dynamics, and problem difficulty: A step toward demystifying tabu search," Journal of Artificial Intelligence, vol. 24, pp. 221-261, 2005 . 